\title{
Correction to: Tango Dance Can Reduce Distress and Insomnia in People with Self-Referred Affective Symptoms
}

\author{
Rosa Pinniger ${ }^{1}$ Einar B. Thorsteinsson ${ }^{1} \cdot$ Rhonda F. Brown ${ }^{2}$. \\ Patricia McKinley ${ }^{3,4}$
}

Published online: 11 December 2019

(c) American Dance Therapy Association 2019

\section{Correction to: Am J Dance Ther (2013) 35:60-77 https://doi.org/10.1007/s10465-012-9141-y}

There were some errors presented in Table 2 in the original article.

Values have been corrected in the version of Table 2 presented here.

The original article can be found online at https://doi.org/10.1007/s10465-012-9141-y.

\section{Rosa Pinniger}

rpinnige@une.edu.au

Einar B. Thorsteinsson

ethorste@une.edu.au

Rhonda F. Brown

rhonda.brown@anu.edu.au

Patricia McKinley

patricia.mckinley@mcgill.ca

1 School of Psychology, University of New England, Armidale, NSW 2351, Australia

2 Australian National University, Canberra, ACT, Australia

3 McGill University, Montreal, QC, Canada

4 Centre Interdisciplinaire de Recherché en Réadaptation du Montréal Metropolitain, Montreal, QC, Canada 
Table 2 Means and standard deviations of dependent variables by groups at pretest, post-test and one-month follow-up (FU)

\begin{tabular}{|c|c|c|c|c|c|c|c|c|}
\hline \multirow[t]{2}{*}{ Variables } & \multicolumn{2}{|c|}{$\begin{array}{l}\text { Meditation } \\
(\mathrm{n}=11)\end{array}$} & \multicolumn{2}{|c|}{$\begin{array}{l}\text { Tango } \\
\left(\mathrm{n}=18^{\mathrm{a}}\right)\end{array}$} & \multicolumn{2}{|c|}{$\begin{array}{l}\text { Exercise } \\
(n=12)\end{array}$} & \multicolumn{2}{|c|}{$\begin{array}{l}\text { Control } \\
(n=23)\end{array}$} \\
\hline & $M$ & $S D$ & $M$ & $S D$ & $M$ & $S D$ & $M$ & $S D$ \\
\hline
\end{tabular}

Life satisfaction

$\begin{array}{lllllllll}\text { Pre } & 15.91 & 4.57 & 15.94 & 7.18 & 16.92 & 8.91 & 18.35 & 7.45 \\ \text { Post } & 20.18 & 3.68 & 22.33 & 4.99 & 19.08 & 8.85 & 18.09 & 7.01 \\ \text { FU } & 22.73 & 3.04 & 20.31 & 7.68 & 19.42 & 8.82 & 17.00 & 7.36\end{array}$

Mindfulness

$\begin{array}{lrrrrrrrr}\text { Pre } & 3.88 & 0.54 & 2.98 & 0.88 & 3.78 & 0.90 & 3.33 & 0.90 \\ \text { Post } & 3.72 & 0.75 & 3.84 & 0.81 & 4.10 & 0.49 & 3.42 & 0.86 \\ \text { FU } & 3.98 & 1.01 & 3.87 & 0.78 & 4.18 & 0.76 & 3.37 & 0.90 \\ \text { Depression } & & & & & & & & \\ \text { Pre } & 10.27 & 5.53 & 12.44 & 5.73 & 10.42 & 3.87 & 9.39 & 5.98 \\ \text { Post } & 6.27 & 4.00 & 6.28 & 3.20 & 6.25 & 3.17 & 9.43 & 6.32 \\ \text { FU } & 6.00 & 4.05 & 5.38 & 3.16 & 6.67 & 4.87 & 9.78 & 6.75\end{array}$

Anxiety

$\begin{array}{lrrrrrrrr}\text { Pre } & 8.00 & 4.92 & 9.28 & 5.04 & 6.75 & 4.88 & 7.65 & 5.76 \\ \text { Post } & 4.91 & 2.91 & 4.94 & 2.88 & 4.00 & 4.51 & 6.74 & 5.58 \\ \text { FU } & 4.36 & 3.44 & 3.88 & 3.01 & 3.58 & 3.09 & 6.70 & 5.72 \\ \text { Stress } & & & & & & & & \\ \text { Pre } & 12.36 & 5.45 & 13.72 & 4.24 & 11.25 & 5.85 & 10.78 & 5.11 \\ \text { Post } & 8.64 & 5.26 & 8.61 & 3.58 & 7.50 & 4.36 & 10.00 & 5.51 \\ \text { FU } & 7.73 & 5.26 & 7.38 & 2.45 & 6.83 & 3.83 & 11.00 & 5.69\end{array}$

Fatigue

$\begin{array}{lllllllll}\text { Pre } & 4.91 & 1.02 & 4.45 & 1.23 & 4.32 & 1.67 & 4.69 & 1.01 \\ \text { Post } & 4.11 & 1.81 & 4.23 & 1.25 & 4.25 & 1.36 & 4.74 & 1.31 \\ \text { FU } & 4.13 & 1.39 & 3.74 & 1.45 & 4.10 & 1.82 & 4.38 & 1.32\end{array}$

Insomnia

$\begin{array}{lllllllll}\text { Pre } & 15.09 & 5.65 & 14.67 & 6.55 & 12.33 & 6.11 & 12.13 & 7.29\end{array}$

$\begin{array}{llllllllll}\text { Post } & 12.00 & 7.77 & 11.11 & 5.14 & 11.25 & 7.86 & 13.39 & 7.06\end{array}$

$\begin{array}{lllllllll}\text { FU } & 9.00 & 5.53 & 8.81 & 6.39 & 9.83 & 7.35 & 12.91 & 6.82\end{array}$

Self-efficacy

$\begin{array}{lllllllll}\text { Pre } & 26.36 & 3.47 & 24.61 & 6.93 & 25.17 & 5.04 & 27.17 & 6.30 \\ \text { Post } & 29.27 & 3.41 & 29.06 & 5.22 & 30.83 & 4.67 & 27.87 & 6.24 \\ \text { FU } & 29.18 & 4.64 & 28.81 & 5.72 & 30.67 & 4.52 & 27.43 & 6.50\end{array}$

Pre before the program, Post at the end of the program, FU one month after the program

${ }^{\text {a }}$ Tango group $\mathrm{n}=18$ at pre- and post-test; and $\mathrm{n}=16$ at the one month follow-up 